\title{
A Lack of Effect on Patient Satisfaction Scores in One Large Urban Emergency Department
}

\author{
Peter W. Crane, Bryce Yerman, Sandra M. Schneider
}

School of Medicine and Dentistry, University of Rochester, Rochester, USA.

Email: sandra_schneider@urmc.rochester.edu

Received September $26^{\text {th }}, 2012$; revised November $9^{\text {th }}$, 2012; accepted November $26^{\text {th }}, 2012$

\begin{abstract}
Background: To deal with emergency department crowding and long waits before patient care, many institutions have placed a doctor in the triage area to initiate treatment and testing. Objective: To determine the effect of a doctor in triage on patient satisfaction scores. Methods: This is an observational study comparing the patient satisfaction scores from days when a physician was in triage to days when a physician was not present. The study was conducted in the ED of an urban academic medical center with excellent primary care resources and payer mix ( $7 \%$ self pay, $11 \%$ Medicaid). Results: There was a mean of 4 (95\% CI 3.1 - 4.5) surveys returned for each day when there was a doctor in triage and a mean of 5 (95\% CI 4.3 - 5.7) surveys for each day without a doctor in triage. Overall satisfaction for the days with a doctor in triage was $79.9 \pm 10.5(95 \%$ CI 77.7, 82.1) compared to $78.8 \pm 9.2(95 \%$ CI $76.5,81.1)(p=0.9)$ on days without a doctor in triage. Conclusion: In this setting, there was no effect of a doctor in triage on patient satisfaction scores. While a doctor in triage may be valuable and cost effective in some settings, it may not provide appropriate benefit in all settings. We suggest that facilities trial a physician in triage program and measure predetermined outcomes such as patient satisfaction scores, length of stay and the percentage of patients left without being seen before investing in additional staffing and cost to sustain such a program.
\end{abstract}

Keywords: Emergency Department Crowding; Triage; Physician in Triage; Patient Satisfaction

\section{Introduction}

Emergency department (ED) crowding, most often caused by the boarding of inpatients in the emergency department, has led to long waits by patients seeking care in the ED. ED waiting times have been increasing $[1,2]$ and a recognized downstream effect of long waiting times is decreased patient satisfaction. It has been previously demonstrated that waiting time and time until seen by a physician/provider are linked with patient satisfaction [3] and timeliness of care was one of the 6 characteristics of quality in the IOM report on quality [4]. The Centers for Medicare and Medicaid Services (CMS) websites such as hosptialcompare.com and other groups place new emphasis on the quality of the patient experience. Hospitals now place increased emphasis on the results of patient satisfaction scores.

Ironically, while hospitals work to improve throughput within the ED, most of the solutions to ED crowding lie outside of the department and require institutional recognition and involvement to accomplish. For example, staff who work in institutions which fail to move boarded patients out of the ED face significant difficulty attaining excellent patient satisfaction scores. Some patients may tie their satisfaction to the timeliness of their bed assignment or movement out of the ED, not to time of their admission decision.

The desire to attain high patient satisfaction scores has led to several innovative, but largely unevaluated, recommendations [5]. One of these recommendations is to place a doctor in triage. Placing a provider in triage reduces the "door to doctor" time and theoretically facilitates patient flow through a department during crowded periods because the provider can order tests in the waiting room or, in some cases, provide care and discharge the patient from the waiting room.

In early September, 2006 our ED instituted a program placing a doctor in triage during the periods of highest occupancy. Our objective of this study is to examine the effect of a doctor in triage on patient satisfaction survey results. We hypothesized that a doctor in triage would decrease wait times and that this would translate into measurably higher patient satisfaction scores as collected by a standard survey. The null hypothesis was that there would be no change in the scores on the days where a doctor was present in triage. 


\section{Methods}

This is a side by side observational study of the effect of a doctor in triage on patient satisfaction scores. PressGaney patient satisfaction surveys were used throughout the study period. Given that improvement of The Press Ganey scores was a top priority for this department, the facility paid for more surveys to be sent to patients. Traditionally the rate of return for these surveys is $<15 \%$ and about 50 surveys are returned in a month. During the time of this study in our institution, with additional surveys sent, approximately 150 - 200 surveys were returned each month. While Press Ganey asks a number of questions regarding satisfaction with nursing, providers and the facility, the question regarding "overall satisfaction" with the visit, scored on a scale of $1-5$, was used for this study. These scores are then turned into a raw score for the overall satisfaction which can be used to compare institutions and to trend within a department.

A faculty physician was assigned to work in the ambulatory triage area in our ED Monday through Friday between the hours of noon and 10pm. Their role was to briefly interview the patient and perform a focused examination on as many patients as possible during triage. In addition they initiated a medical evaluation (ordering labs, Xrays, etc.). Because there was a concern about placing intravenous access in this largely unsupervised population in the waiting room, pain medications were oral (including fentanyl) or intramuscular. Difficulty with staffing led to the decision to not place a doctor in triage on the weekends, even though the total number of patients seen on all days was similar. Further, there were many shifts throughout this initial start up period when staffing prevented a doctor in triage or limited the number of hours the position was staffed. During those times, triage was performed by nursing as had been standard practice. Our state law prohibits nurses from using standing orders or advanced triage protocols so triage nurses were unable to initiate testing.

The study period was $1 / 10 / 06-3 / 27 / 06$. The physician in triage program had been initiated in early September so that the program was running smoothly by the start of the study period. Nonetheless, because this program called for increasing the staffing each day, many shifts went unfilled. The physician in triage saw only ambulatory adult patients as there are geographically distinct areas for pediatric and ambulance triage. Door to doctor time was captured by the electronic tracking system (IBEX).

Returned Press Ganey surveys were coded by the date of service. Only those returned from adult patients were used for this study. It was decided a priori to exclude any day when a doctor was present for less than 4 hours and those days when no Press Ganey surveys were received. The Press Ganey surveys received on the remaining days were divided into 2 groups based on the presence or absence of a doctor in triage. The raw score for overall satisfaction was compared using a student $t$ test with significance set at $\mathrm{p}<0.05$.

This study was performed in a tertiary care center with an emergency medicine residency program that during this period had a volume of 95,000 patients per year. About $78 \%$ of adult patients are treated and release with an approximate $22 \%$ admission rate. Payer distribution is $14 \%$ MC, $11 \%$ MA, $59 \%$ private insurance and $7 \%$ self pay. Our patient population is approximately $63 \%$ White, 31\% Black or African American, 1\% Asian. We do not gather data for ethnicity.

The study was approved by the Research Subject Review Board of the University of Rochester Medical Center.

\section{Results}

There were 84 days that met the criteria for a doctor in triage and 59 days where there was no doctor in triage. Median door to doctor time between $12 \mathrm{p}$ and $10 \mathrm{p}$ on days with a doctor in triage was $7 \pm 39.1$ minutes and on the days without a doctor in triage median time was $65 \pm$ 84.4 minutes. There was a mean of 4 (95\% CI 3.1 - 4.5) surveys returned for each day when there was a doctor in triage and a mean of 5 (95\% CI 4.3 - 5.7) surveys for each day without a doctor in triage.

Overall satisfaction for the days with a doctor in triage was $79.9 \pm 10.5(95 \%$ CI $77.7,82.1)$ compared to $78.8 \pm$ $9.2(95 \%$ CI $76.5,81.1)(\mathrm{p}=0.9)$ on days without a doctor in triage.

\section{Discussion}

In this study, placing a physician in triage did not improve patient satisfaction scores.

Previous studies have demonstrated that a physician in triage decreases door to doctor time, reduces length of stay and also lowers the number of patients who leave without being seen [6,7]. Several case studies have been published citing impressive improvement in patient satisfaction scores after initiation of a physician in triage program $[8,9]$. Other studies have demonstrated that decreased door to doctor time correlates with improved patient satisfaction [3].

Physician in triage programs have been shown to be effective in several studies [6]. However many of these studies were done in public hospitals where larger numbers of patients with minor illnesses can be treated and discharged by the doctor in triage. Our area is known for its access to primary care with $85 \%-90 \%$ of all adult patients having a primary care provider in periodic surveys [10]. Our patients make few ED visits for relatively minor illnesses and even those who are eventually dis- 
charged generally require some advanced testing.

As in other work, our study showed that placing a doctor in triage decreases door to doctor time. However in this study it did not translate into higher patient satisfaction scores, one of the primary goals of the program. It is possible that patients did not recognize that the doctor in triage was a provider. Though all physicians were instructed to introduce themselves, given the brief encounter in a location traditionally staffed only by nurses, patients may have not realized they were being evaluated by a doctor.

We do believe that reducing wait times correlates with patient satisfaction. It may be that reducing only a single component of that wait is not perceived as significant in our patient population. There are other options available to decrease wait times that may work better in selected situations. Some hospitals have applied modern business assessment tools such as LEAN or Six-Sigma, and others have utilized standing orders completed by nurses at triage or initial assessment $[11,12]$. Still other hospitals have increased physician staffing, but in the formal care area rather than the triage area. Given the cost associated with staffing a physician in triage, some hospitals may find it prudent to implement other more high-yield solutions in order to improve patient satisfaction at a better cost versus benefit ratio. When locally developed and implemented, such tools may provide sustained improvement in patient satisfaction without the cost associated with addition providers at triage.

\section{Limitations}

This study was performed in a single emergency department with demographics more typical of a community hospital than a public hospital. Therefore, these findings may not be typical of all institutions. The Press Ganey survey is not the only survey tool available and it is possible that other more sensitive tools might perform differently. The Press Ganey survey system has been criticized by many, however it remains one of the most widely used systems for assessing patient satisfaction. Generally the results of these surveys are made from a small number of those returned (typically 50 per month) and only surveys returned from discharged adult patients are used. In addition, patients who have been seen in the previous 90 days and those who leave without being seen are not eligible for this survey. In our study, additional surveys were purchased to assess this program and our individual physician performance. The effect of a doctor in triage may have been too small to be reflected in the Press Ganey tool. However, this is one of the standard tools used to measure patient satisfaction and any benefit derived from placing a physician in triage must be measured by this tool in order to be recognized.
Door to doctor time was recorded by the electronic tracking system. The system requires the provider to "sign up" for the patient in order to begin time tracking. It is possible that this time may not have been the exact time of initial patient encounter. Regardless, the significant difference between the days with and without a triage doctor is not likely to be related to time-entry error.

\section{Conclusion}

We found no change in the Press Ganey patient satisfaction scores during periods of physician at triage patient encounters when compared to periods when there was no doctor in triage present. While this program may be valuable and cost effective in some settings, it may not provide appropriate benefit in all settings. We suggest that facilities trial a physician in triage program and measure predetermined outcomes such as patient satisfaction scores, length of stay and the percentage of patients left without being seen before investing in additional staffing and cost to sustain such a program.

\section{REFERENCES}

[1] R. Ding, M. L. McCarthy, J. S. Desmond, et al., “Characterizing Waiting Room Time, Treatment Time, and Boarding Time in the Emergency Department Using Quantile Regression,” Academic Emergency Medicine, Vol. 17, No. 8, 2010, pp. 813-23. doi:10.1111/j.1553-2712.2010.00812.x

[2] L. I. Horwitz, J. Green and E. H. Bradley, "US Emergency Department Performance on Wait Time and Length of Visit,” Academic Emergency Medicine, Vol. 55, No. 2, 2010, pp. 133-141.

[3] B. Bursh, J. Beezy and R. Shaw, "Emergency Department Satisfaction: What Matters Most," Academic Emergency Medicine, Vol. 22, No. 3, 1993, pp. 586-591. doi:10.1016/S0196-0644(05)81947-X

[4] IOM Committee on Quality of Health Care in America, "Crossing the Quality Chasm: A New Health System for the 21st Century,” National Academies Press, Washington DC, 2001.

[5] T. A. Bruce, J. M. Bowman and S. T. Brown, "Factors That Influence Patient Satisfaction in the Emergency Department,” Journal of Nursing Care Quality, Vol. 13, No. 2, 1998, pp. 31-37. doi:10.1097/00001786-199812000-00005

[6] B. H. Rowe, X. Guo, C. Villa-Roel, et al., "The Role of Triage Liaison Physicians on Mitigating Overcrowding in Emergency Departments: A Systematic Review,” Academic Emergency Medicine, Vol. 18, No. 2, 2011, pp. 111112. doi:10.1111/j.1553-2712.2010.00984.x

[7] T. C. Chan, J. P. Killeen, D. Kelly and D. A. Guss, "Impact of Rapid Entry and Accelerated Care at Triage on Reducing Emergency Department Patient Wait Times, Lengths of Stay, and Rate of Left without Being Seen," Annals of Emergency Medicine, Vol. 46, No. 6, 2005, pp. 
491-497. doi:10.1016/j.annemergmed.2005.06.013

[8] “Care Initiation Area Yields Dramatic Results,” ED Management, Vol. 21, No. 3, 2009, pp. 28-29.

[9] “Competitive Concerns Bring PAs, NPs into Triage Area," ED Management, Vol. 20, No. 6, 2008, pp. 67-68.

[10] M. F. Kamali, A. Jain, M. Jain and S. M. Schneider, "Patient with Access to Primary Care Call Their Doctors before Coming to the Emergency Department with Lower Acuity Illness," Annals of Emergency Medicine, Vol. 58, No. 4, 2011, p. S234.

doi:10.1016/j.annemergmed.2011.06.196
[11] R. W. Rosmulder, J. J. Krabbendam, A. H. Kerkhoff, et al., “'Advanced Triage' Improves Patient Flow in the Emergency Department without Affecting the Quality of Care,” Nederlands Tijdschrift Voor Geneeskunde, Vol. 154, 2010, p. A1109.

[12] D. Ng, G. Vail, S. Thomas and N. Schmidt, “Applying the Lean Principles of the Toyota Production System to Reduce Wait Times in the Emergency Department," Canadian Journal of Emergency Medicine, Vol. 12, No. 1, 2010, pp. 50-57. 